\title{
WHAT CAN BE LEARNT FROM 130 CHILDREN'S DREAM WHEELCHAIR DESIGNS? ELICITING CHILD-CENTRED INSIGHTS USING AN INTERDISCIPLINARY DESIGN ANALYSIS FRAMEWORK
}

\author{
O'Sullivan, Cara \\ Nickpour, Farnaz \\ Bernardi, Francesca \\ University of Liverpool
}

\begin{abstract}
The design of inclusive paediatric mobility (IPM) interventions, such as children's wheelchairs, are entangled with technological, health and social considerations. As narratives around childhood, disability and mobility shift and transform, these entanglements evolve. In order to optimise the experience of childhood mobility, IPM designers must understand and respond to such changes and channel children's own requirements, desires and 'dreams' into the design process; this can be achieved by utilising a child-centred design approach. This paper identifies meaningful child-centred IPM design insights and opportunities through the interdisciplinary analysis of 130 dream wheelchair designs by disabled children, aged 4 to 17 years. A novel interdisciplinary and child-centred design analysis framework is developed to dissect, categorise and code the topics and features expressed through visuals and written descriptions in each of the children's dream wheelchair designs. Children's mobility narratives, desires and requirements are elicited and trending topics are discussed. It is proposed that valuing children's voices in the IPM design process could alter both the process of designing IPM interventions as well as the product outcomes.
\end{abstract}

Keywords: Inclusive design, Paediatric Mobility, User centred design, Childhood, Collaborative design

\section{Contact:}

O'Sullivan, Cara

University of Liverpool

Industrial Design

United Kingdom

cara1@liverpool.ac.uk

Cite this article: O’Sullivan, C., Nickpour, F., Bernardi, F. (2021) 'What Can Be Learnt from 130 Children's Dream Wheelchair Designs? Eliciting Child-Centred Insights Using an Interdisciplinary Design Analysis Framework', in Proceedings of the International Conference on Engineering Design (ICED21), Gothenburg, Sweden, 16-20 August 2021. DOI:10.1017/pds.2021.602 


\section{INTRODUCTION}

Lack of child-centred design approach has historically resulted in various issues around the desirability, feasibility and usability of inclusive paediatric mobility (IPM) interventions such as wheelchairs (O'Sullivan and Nickpour, 2020). The multidisciplinary nature of the IPM field requires various stakeholder voices to be captured and considered in the design process, which has often resulted in children's voices being diluted, repressed or sometimes excluded. To address such issues and ensure the future of IPM design is built upon a holistically considered and inclusive foundation, clarity around why and how to capture, interpret, and represent the voices of children needs to be improved. The word 'voice' is used metaphorically in the context of this paper to signify opinions and expressions encapsulated by all modes of communication, with explicit inclusion of non-verbal mediums. Children are lived experience experts in their own mobility and so their perceptions, opinions and ideas around their mobility are of significant value in an IPM design process. Incorporating children's voices, perspectives, requirements, and rights, centrally in the design process can elevate the status of their interests and views (Can \& İnalhan, 2017), psychologically and physically empower them (Hussain, 2010), better understand user experiences (Desmet \& Dijkhuis, 2003) and reveal unmet requirements and desires (Yamada-Rice, 2019). In the context of IPM design, this could uncover currently unacknowledged, unstated and unmet narratives, requirements, and challenges around children's mobility, and identify child-centred design opportunities to improve the desirability, functionality and usability of interventional outcomes. The urgency to involve children with IPM design stems from the understanding that until IPM interventions fulfil the requirements and desires of children, their experience of childhood will be less than optimal and their right to achieve the "fullest possible social integration and individual development" may not be fulfilled (United Nations, 1990).

\section{RESEARCH AIMS AND METHODOLOGY}

This paper sets out to identify what could be captured through children's voices in an IPM design process, both qualitatively and quantitatively, when children take on the active role as Designer. The paper also explores how interdisciplinarity can help extract meaningful insights from children's design expressions. To address these two aims, a qualitative secondary dataset was selected for interrogation and analysis, in which 130 children who use a wheelchair designed their 'dream wheelchairs'. This paper analyses the children's responses and collates their imagined mobility futurescapes, ideas, and dreams, in order to unpick and assess hierarchy of narratives, requirements, and challenges around children's wheelchairs. Recognising the complex nature of inviting and including children's design input, the analysis embraces an interdisciplinary approach which brings together researchers and IPM stakeholders with distinct experiential and disciplinary backgrounds, to interweave their differing lenses throughout the process. The questioning and merging of different disciplinary perspectives can provide a more holistic, rigorous and exhaustive approach to child-centred research which in turn ensures children's voices are deeply considered without the distinctions of disciplinary bias, and with reduced chance of misinterpretation (CohenMiller \& Pate, 2019).

\section{ANALYSING CHILDREN'S DREAM WHEELCHAIR DESIGNS}

\subsection{Data Collection, Context and Significance}

The selected secondary dataset was collected through a national design competition which asked children from across the United Kingdom who use a wheelchair, to design their 'Dream Wheelchair'. The competition aimed to create a national conversation involving the voices and ideas of as many young wheelchair users as possible, and ran for seven weeks in 2019; it encouraged participants to visualise or describe their designs either individually, with assistance, or in a group. Dream wheelchair designs were submitted by 130 wheelchair users aged between four to seventeen years, making this the largest and most recent qualitative dataset of IPM designs by children. The dataset aligns with the research aims set out in this paper whilst being adopted for a purpose different to the original intentions of the data collection (Spurlock, 2020). The author of this paper is responsible for leading the analysis of the dataset and has no connection to any of the competition entrants, nor any involvement with organising the competition. 


\subsection{Child-centred Design Analysis Framework}

To ensure all aspects of the children's designs could be captured systematically for discussion on multiple levels, an interdisciplinary theoretical framework was devised to provide structure in the analysis process. Whilst various tools and frameworks exist to facilitate the analysis of designs, no such thing exists to facilitate holistic interdisciplinary analysis of designs from a child-centred perspective. Several existing frameworks were identified as points of reference from across disciplines, and synthesised keeping 'theories separate but integrated' (CohenMiller \& Pate, 2019) to create the underpinnings of a holistic intersecting framework suitable for this purpose. The conceptual 'Three Facets of Language and Art framework' (Barroqueiro, 2010) was used as the spine of this framework to divide the analysis into references relating to the 'Content' and 'Context' of children's designs, and the 'Format' they used to express their design. The content segment of the framework was expanded by incorporating 'Categories for Reasons a Possession Is Valued' (Richins, 1994), 'Hierarchy of User Needs' categories (Walter, 2011), and foundational Industrial Design principles including 'CMF', form, and metaphor. The context segment of the framework was made up from aspects of the 'AEIOU' tool (Robinson, 2015) and the PESTLE analysis tool (Oxford, 2016). The format segment of the framework was included to record the child's communication medium (e.g. a drawing or written description of their design), as well as who else helped to design it, if specified. The framework was then further detailed and refined by a range of IPM stakeholders (i.e. lived experience experts - children and parents, occupational therapists, IPM designers and childhood researchers) through two workshops which invited each of them to review a sample of 14 randomly selected designs from the dataset. The aim of this was to identify high level themes, topics or points of interest which were recorded and mapped onto the framework. This resulted in the addition of new nodes, alterations to the order of nodes, language adjustments, and rearrangement of the visual structure of the framework. Figure 1 illustrates which stakeholders influenced the additional nodes included in the framework.

\section{CHILD-CENTRED DESIGN ANALYSIS FRAMEWORK}
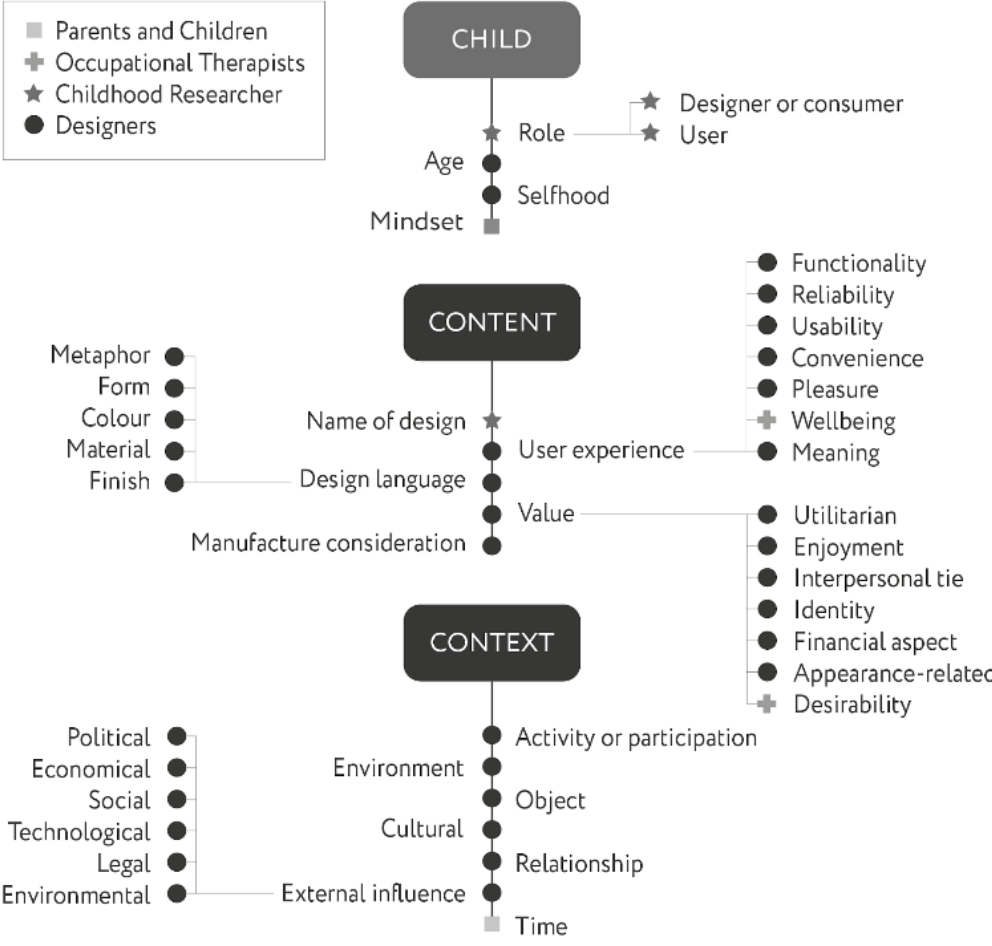

FORMAT

Communication medium

Whose voice/s

Figure 1. The refined child-centred design analysis framework. 


\subsection{Coding Dataset Using the Framework}

The refined child-centred design analysis framework (Figure 1) was then 'translated' into a coding structure using NVivo software to code, categorise and begin to analyse features and functionality expressed through the dataset. This coding practice revealed child-centred insights and trends, as well as different disciplinary and stakeholder perspectives. The 'Child' and 'Format' segments of the framework were used to gain a better understanding of the child's influences, approach and nature of engagement with the activity. These segments have been excluded from this paper due to not being immediately relevant to the research aims, however, they have been included in Figure 1 to provide a complete visualisation of the analysis framework, and should be further explored in future research.

\section{FINDINGS FROM DREAM WHEELCHAIR ANALYSIS}

The richness and diversity of children's drawings and texts enable different levels of analysis with two distinct potential design research outcomes. On one level, children's dream wheelchair designs can help capture specific user requirements and product features, informing the design of paediatric wheelchairs through collecting URS (User Requirements Specifications) and PDS (Product Design Specifications). In this capacity, such analysis is aimed at 'problem solving', and is recognised as a 'Research For Design' activity. On the other level, the designs could help uncover children's highlevel narratives, social imaginaries, and meanings around childhood, mobility and disability. Rather than interpreting children's input based primarily on feasibility, their designs could be unpicked, analysed and used to identify what is missing from the current state of IPM interventions from a childcentred perspective. In this capacity, the analysis is aimed at 'problem framing', and recognised as a 'Research Through Design' activity.

The collective magnitude and hierarchy of topics expressed by children in their dream wheelchair designs can be gauged by taking a closer look at trends from the analysis results in Figure 2 and Figure 3. Of the many contextual variables identified through analysing children's designs, age proved to be the most notable influencer for variations in the expressed topics (based on the available data). Age has thus been used as a marker for comparative analysis by splitting all the results into designs by 4-12 year olds ( 70 children), and designs by 13-17 year olds (60 children) to highlight topics which vary notably between the age groups.

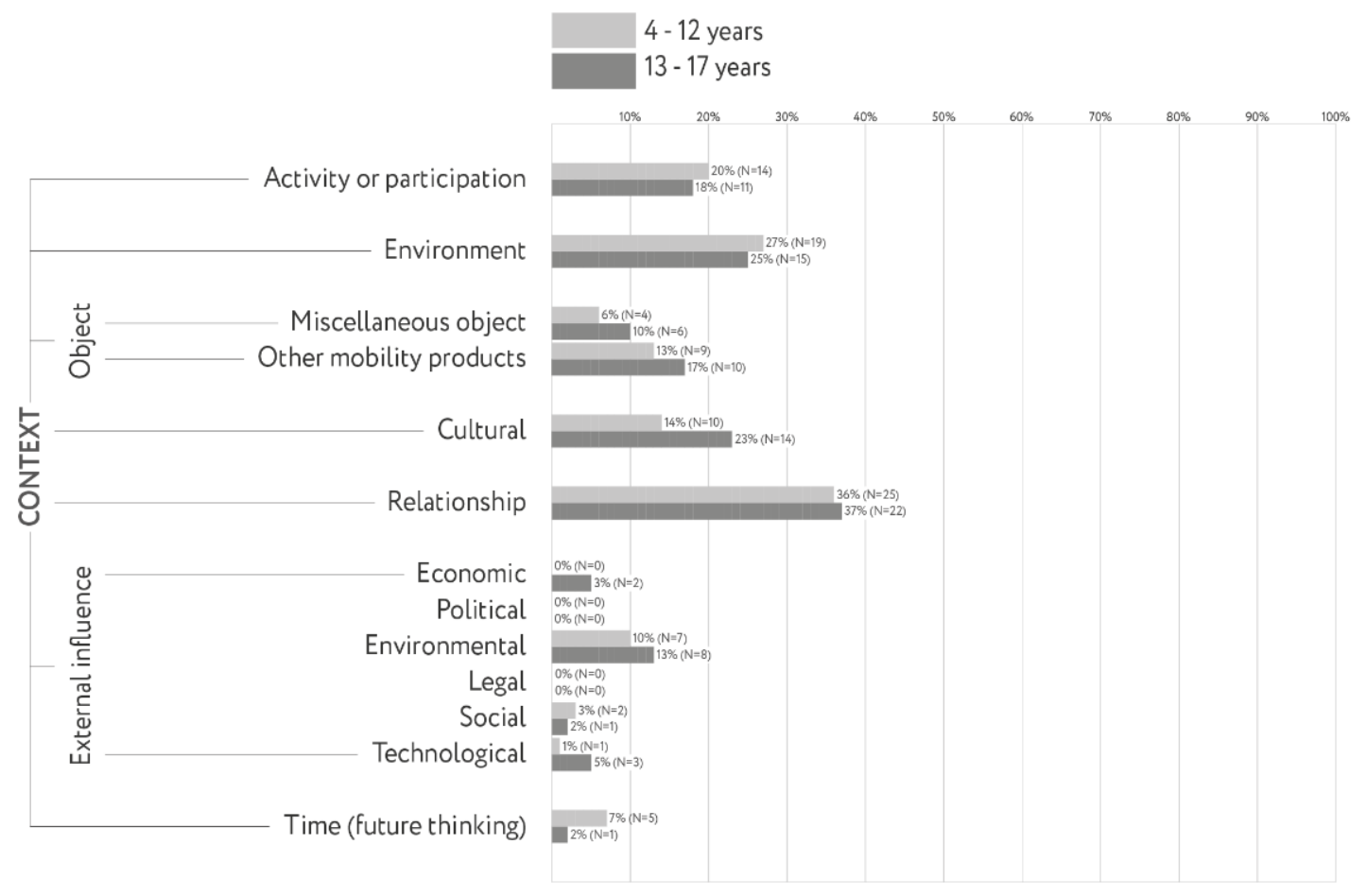

Figure 2. Results from dream wheelchair design analysis; Context segment of framework. 


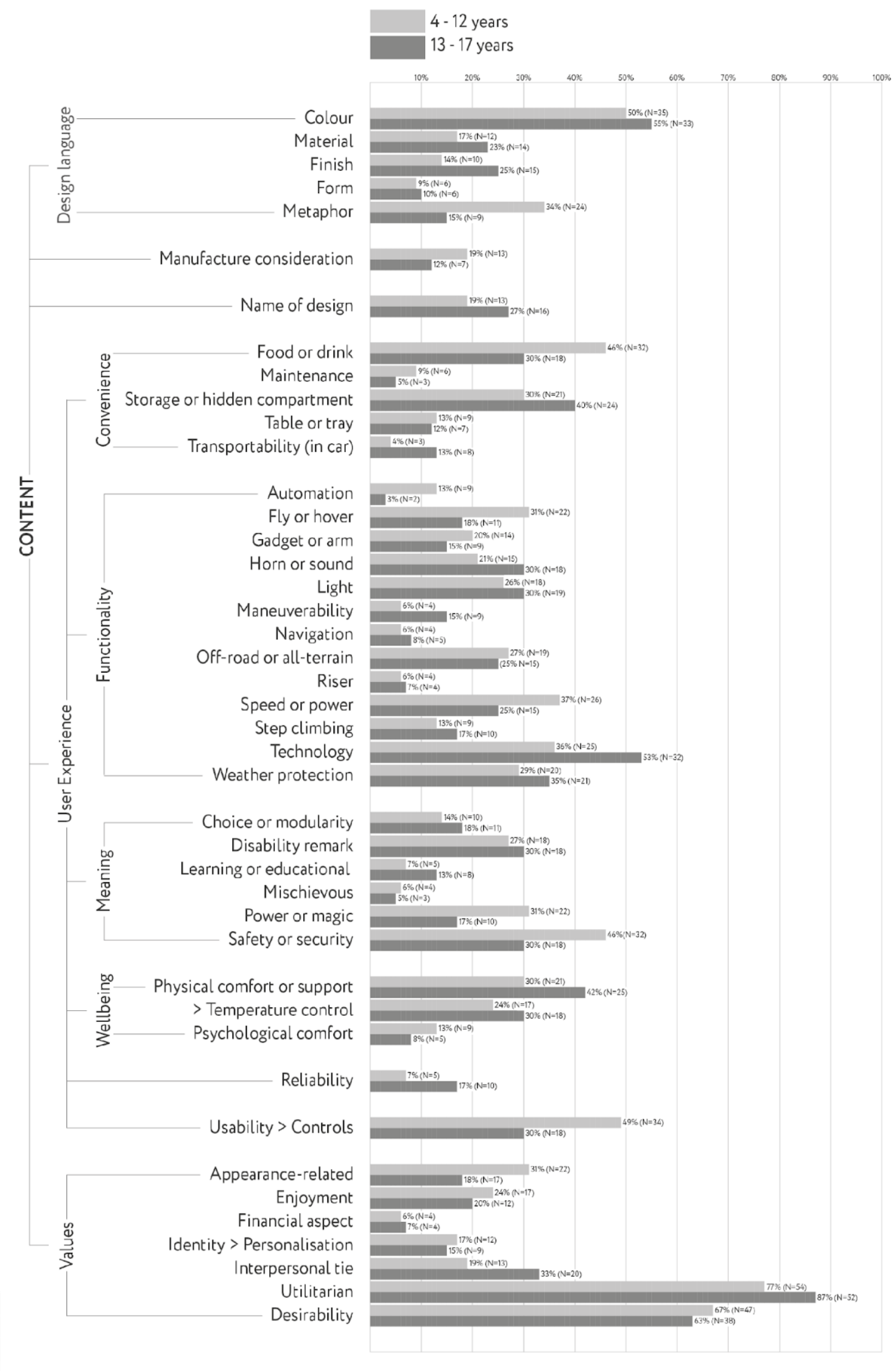

Figure 3. Results from dream wheelchair design analysis; Content segment of framework. 


\section{TRENDING TOPICS AND INITIAL INSIGHTS}

The most widely referenced topics in the analysis are classified as 'trending' and include all which were referenced by $30 \%$ or more of children within either age group. Sections 5.1 to 5.8 offer further insights into the children's designs and imagined mobility scenarios by discussing the trending topics and commenting on their significance, including variations in trend rates between the different age groups.

\subsection{Trending in 'Values'}

\subsubsection{Utilitarian}

Trending at $82 \%$ overall, utilitarian expressions were the most referenced topic from the analysis, captured in $77 \%$ of designs by children aged 4 to 12 years, and in $87 \%$ of designs by children between 13 to 17 years. Utilitarian expressions encompass a broad range of subtopics relating to functionality, expressed both implicitly and explicitly. Examples include "the tyres should be thick, chunky and solid so there is no risk of punctures" and "the chair is fun and relaxing but most importantly functional". This trend indicates that children have considered the functionality of their wheelchair in more detail than the visual aspects of their wheelchair which could signify an unspoken hierarchy of importance.

\subsubsection{Desirability}

The second most referenced topic in children's designs was desirability, with overall $65 \%$ of children emphasising the need for their wheelchair to appeal to their personal aspirations or visual preferences, at the same time as seeking respect and sometimes envy from others. There is little variation between age groups around this topic, with references by $67 \%$ of children aged 4 to 12 years, and by $28 \%$ of children between 13 to 17 years. Incremental innovations captured at this topic tended to focus on improving the desirability of their existing wheelchair through cosmetic alterations or the addition of accessories. More radical innovations around desirability tended to focus on re-imagining what the entire wheelchair concept could enable, drawing on fantasy and fiction worlds, and indulging in their dreams to redefine what is possible.

\subsubsection{Appearance related}

Trending at $30 \%$, references which attributed value to appearance can be found in $31 \%$ of designs by children aged 4 to 12 years, and in $28 \%$ of those by children between 13 to 17 years. References to this topic state the worth or importance of appearance which differentiates the topic to appearance related references within the 'Design Language' section of the framework. References to this topic cover how the user appears in their wheelchair, how others perceive the wheelchair, why they want their wheelchair to appear in a certain way, how certain colours or styles make them feel, and what their visual choices represent or mean. One example states "I put a lot of colours on my wheelchair so that it looks like a rainbow and makes me feel happy every day."

\subsubsection{Interpersonal ties}

Interpersonal ties were trending at $25 \%$ overall, appearing in $19 \%$ of designs by children aged 4 to 12 years, and in $33 \%$ of designs by children between 13 to 17 years. Many children make explicit reference to the value of companionship through designing considerate accessories or features to be used by, or engage with, others in ways that existing wheelchairs do not. References to the value or meaning of children's interpersonal ties include examples such as: "... an extra seat attached so that you can take your carer on long journeys", “...dog trailer means that my dog can be with me anytime, anywhere", and a toy machine "so I can give toys to all the children that I see". By seeking to include others through these design features, it is possible to see that the children are considering different ways to induce equality and shared participation in their wheelchair experience.

\subsection{Trending in 'Design Language'}

\subsubsection{Colours}

References to colour/s were annotated or described with detail in $52 \%$ of the children's designs. This topic has little variation across age groups, with reference by $50 \%$ of children aged 4 to 12 years and $55 \%$ of children between 13 to 17 years. The most common areas of the wheelchair where colour is 
referred to include: the seat, the frame, the wheels, the controls (e.g. buttons) and accessories (e.g. colour changing lights). Through analysing the nature of children's expressions, references in this topic generally come across as desirables rather than requirements.

\subsubsection{Metaphor}

Overall $25 \%$ of children use metaphors to describe their designs, however, there is a noteworthy difference between the use of metaphors across the two age groups; $34 \%$ of children aged 4 to 12 years use a metaphor whilst only $15 \%$ of children between 13 to 17 years use one. This correlates closely to the trends in imagination-rich topics (mentioned later in this paper) such as 'Power or Magic' and could be attributed to older children approaching the design task more rationally or possibly with a wider vocabulary and thus not requiring such imaginative language to communicate their concepts. Examples of metaphors used in children's designs include: "as powerful as a crocodile", "as comfortable as a couch", "like a spa on the move", "it will feel like my mum and look after me for forever."

\subsection{Trending in 'Functionality'}

\subsubsection{Technology}

With $44 \%$ of children including references to technology in their designs, this is the highest overall trending topic in the 'Functionality' section of the analysis framework. This trend increases for children over the age of 12, with technology appearing in 36\% of designs by children aged 4 to 12 years whilst appearing in 53\% of designs by children between 13 to 17 years. Designs by those aged 4 to 12 years mostly relate to enjoyment, usability and convenience, whilst designs by those between 13 to 17 years also relate to external technological influences and the user's relationships. The majority of references to tech equipment relate to iPads, TVs, mobile phones and charging devices.

\subsubsection{Weather protection}

With $32 \%$ of children including references to weather protection features. This topic has a slight increase in trend rate with age, with $29 \%$ of children aged 4 to 12 years, and $35 \%$ of children between 13 to 17 years including weather protection features in their designs. Overall, $93 \%$ of these children specifically refer to protection from various types of precipitation (i.e. snow, rain, hail). Protection from sunshine is also a dominant theme within this topic.

\subsubsection{Speed or Power}

References to speed or power are captured in $32 \%$ of all children's dream wheelchairs. The topic trends higher in designs by children aged 4 to 12 years, with references by $37 \%$, compared to $25 \%$ in designs by children between 13 to 17 years. The dominant underlying narrative accompanying references in this topic is a desire to move fast, and in many cases to move faster than able-bodied peers. Reasoning behind one of the designs referencing speed states "when my friends are running I feel really left out, even when they stay with me I would like to be racing but I cannot." Another writes "A booster pack for winning first at Sports Day!"

\subsubsection{Lights}

Lights were trending at $28 \%$ overall, appearing in $26 \%$ of designs by children aged 4 to 12 years and appearing in $32 \%$ of designs by children between 13 to 17 years. Lights were predominantly mentioned as either a safety feature, a functional appearance related feature, or both. Words used when referencing lights as a safety feature included: automatic, dark, flashing warning, headlights, indicators, night time, reflectors. Words used when referencing lights as a functional appearance related feature included: a chandelier inside the wheelchair, colour changing, disco, fairy lights, LED wheels, multi-coloured lights, spotlights.

\subsubsection{Fly or Hover}

Trending at $25 \%$ overall, reference to flying or hovering functions were made in $31 \%$ of designs by children aged 4 to 12 years and in $18 \%$ of designs by children between 13 to 17 years. The drop in trend rate for children over 12 years could be a result of older children giving greater consideration to 
the viability or feasibility of their designs than younger children. Although the concept of a flying wheelchair might seem unviable, it may be possible to unpick children's expressions to reveal deeper or alternative narratives with viable reasoning. For example, $27 \%$ of these designs refer to using a fly or hover function specifically as a means to overcome steps or rough terrain in their wheelchair.

\subsubsection{Horn or Sound}

References to a horn or sound are captured in $25 \%$ of all children's dream wheelchairs. The topic trends higher in designs by children between 13 to 17 years, with references by $30 \%$ of them, compared to only $21 \%$ of children aged 4 to 12 years. The two dominant narratives attached to references in this topic are safety and entertainment. 55\% of references in this topic refer to a horn or sound function for the purpose of safety, such as a warning beep, whilst the other $45 \%$ refer to sound as a means for entertainment.

\subsection{Trending in 'Usability'}

\subsubsection{Controls}

Trending at $40 \%$ overall, details about the nature of wheelchair controls were captured in $49 \%$ of designs by children aged 4 to 12 years, and in $30 \%$ of those by children between 13 to 17 years. All descriptions of control function adopt a narrative of facilitating usability or independence for the user. Specific types of controls mentioned include: Buttons, chin controls, control panels, cruise control, eye gaze, fingerprint recognition, gear sticks, handlebars, hand controls, head controls, knobs, levers, manual and automated joysticks, mind control, remote controls, steering wheels, switches, touch screen, triggers, various types of brakes, voice recognition, and wheelchairs which can be controlled by customisable control inputs based on the users' choice.

\subsection{Trending in 'Meaning'}

\subsubsection{Safety or Security}

Of all children, $38 \%$ expressed the inclusion of safety or security features in their dream wheelchair designs. Reference to such features declines for children over the age of 12 , with safety or security features expressed in $30 \%$ of designs by children between 13 to 17 years whilst being expressed in $46 \%$ of designs by children aged 4 to 12 years. Keywords used in reference to this topic include: 999 call if I need help, able to eject me, alarm, anti-tip, automatic cushion if you fall out, automatic seatbelt, beeper to warn people, brake pedal, call button connected to parents, crash prevention, emergency button, fingerprint recognition, first aid kit, glow in the dark, handles, harness, helmet visor, keys to open, life jacket under seat, panic button, parachute, police light, protection, repair kit, ring for help, safety clips, safety lights, secure storage, sensors, shield, SOS signal button, winch under seat if I get stuck in mud.

\subsubsection{Disability Remark}

Of all children, 28\% expressed some kind of account or remark relating to their lived experience with a disability, or the behaviours of those around them as a wheelchair user. A slight increase in references can be seen with age as $26 \%$ of children aged 4 to 12 years make a disability remark in their design, whilst $30 \%$ of children between 13 to 17 years do so. These references emerged primarily as written descriptions and have in many cases been embodied by features which address their remark. Examples of such include: "does more than serve as an alternative to walking", "You won't get the sympathy stare...you will get a WHOA, look at that chair", "the height can be adjusted so you can look down on others for a change", "an air horn for people who reach over me", and "Most restaurants and places do not have wheelchair accessible tables so you could use your own".

\subsubsection{Power or Magic}

Trending at $25 \%$ overall, references to power or magic occur in $31 \%$ of designs by children aged 4 to 12 years, and $17 \%$ of designs by children between 13 to 17 years. The dominant underlying narrative in this topic centres on children's abundance of imagination, detailing the adventures they would go on and powers they would have if they could really live their dreams. References to such magical fantasy lands include: "a portal to your own secret dimension" and "a time travel button that takes you to the past". Another common underlying narrative in this topic is for the wheelchair to not only enable their mobility impairment to turn from disability into ability, but to grant them abilities superior to their able-bodied peers. 


\subsection{Trending in 'Convenience'}

\subsubsection{Food or Drink}

With an equal trend rate to Safety and Security, $38 \%$ of children included references to food or drink in their designs, making it the highest overall trending topic in the 'Convenience' section of User Experience within the analysis framework. This trend was higher for children aged 4 to 12 years, with $46 \%$ of them referencing food or drink in their wheelchair design compared with $30 \%$ of children between 13 to 17 years. References to food and drink varied broadly and included: Trays, storage, coolers, fridges and baskets specifically for food or drink, ways to cook food on the wheelchair, various kinds of built-in drinks machines, supplies or machines to create food, food dispensers, methods for sharing food with others, emergency food supplies, secret stashes, wheelchairs powered by food waste, and wheelchairs made out of food.

\subsubsection{Storage or Hidden Compartment}

References to storage or hidden compartments were found in $35 \%$ of all children's designs and this was the highest trending topic for children between 13 to 17 years in the 'Convenience' section of User Experience within the analysis framework. Of children aged 4 to 12 years, 30\% designed storage or hidden compartments intended for items including: accessories, assistant/carer's belongings, bags, books, chocolate, dog leads, drinks, emergency equipment, food, litter, remote control, snacks, sweets, technology, and toys. Of children between 13 to 17 years, $40 \%$ designed storage or hidden compartments specifically for items including: bibs, bits and bobs, cups, glasses, jewellery, pens, phone, rain cover, SatNav, snacks, spare batteries, spare wheel, suitcase, table, walking sticks, wallet, and water bottles.

\subsection{Trending in 'Wellbeing'}

\subsubsection{Physical Comfort}

Trending at $35 \%$, descriptions relating to physical comfort were captured in $30 \%$ of designs by children aged 4 to 12 years, and in $42 \%$ of those by children between 13 to 17 years. Physical comfort could be considered as a minimum requirement in the design of a dream wheelchair, and may have been considered a given by the $65 \%$ of children who did not explicitly refer to it in their designs. The main touchpoint associated with comfort was the seat of the wheelchair, many of which also used terminology specifically associated with seating systems. Reoccurring keywords in the analysis included: aches, back, bed, bottom, calm, comfort, comfortable, comfy, cushion, ergonomic seat, footrest, head rest, lie down, low energy, massage, moulded shape, padding, pain, position, postural support, recline, riser, shock absorbers, sleep, smooth ride, sore, spa, springs, stress relief, suspension, tilt in space, tired.

\subsubsection{Temperature Control}

Temperature control trends at $27 \%$ overall, with references from $24 \%$ of children aged 4 to 12 years, and from $30 \%$ of children between 13 to 17 years. Features regarding only heating or keeping warm account for $77 \%$ of these references, those regarding only staying cool account for $9 \%$, and both heating and cooling functions account for $14 \%$. The majority of references mention weather conditions as the reason for needing such function. Contextual factors which might influence this result include the time of year and weather when the child designed their dream wheelchair, as well as the typical year-round climate and weather where they live, geographically speaking.

\subsection{Trending in 'Context'}

\subsubsection{Relationship}

At $36 \%$, the only 'trending' topic within the Context section of the analysis framework was 'Relationship' which encompasses references to anyone or anything the user expresses a connection or interpersonal interaction with. This topic has little variation across age groups, with references by $36 \%$ of children aged 4 to 12 years, and $37 \%$ by children between 13 to 17 years. This topic relates closely to 'interpersonal ties' but references do not necessarily state the nature, worth or importance of the expression. References commonly relate to participating in activities with others, functions which make the wheelchair serve others as well as the user, and disability remarks around the way the user interacts with or compares to others. Children aged 4 to 12 years also commonly relate to relationships with pets 
or toys. Such consideration for others highlights children's desires to explore their social role beyond the functional aspects of mobility, signalling their concern for the wellness and participation of others in ways that dismantle a rhetoric of need and dependency, hinting towards the potential for a wheelchair to facilitate child-initiated socialisation through empowering other (potentially able-bodied) people.

\section{CONCLUSION \& FURTHER RESEARCH}

This paper analysed 130 children's dream wheelchair designs to explore what could be captured by involving their voices in an IPM design process. A novel interdisciplinary and child-centred framework was developed to facilitate the analysis of children's visual or written design input in order to extract, analyse and quantify meaningful insights. The framework could be utilised by other domains both closely related and more distant from IPM, to help elicit and elevate children's voices in design processes. Initial findings from the analysis identified trending topics and uncovered children's mobility narratives, requirements, and imagined futurescapes, which could be implemented by IPM practitioners and researchers to inform and steer strategy, design and development of future child-centred mobility solutions. These initial findings will be further analysed to connect findings to research in the fields of emotional and experience design and to make sense of children's contributions beyond the most widely referenced topics within different age groups.

Future research will address the design analysis framework as a whole, with specific focus on the 'Child' and 'Format' segments, to explore how engagements with children in the IPM design process could be optimised. Questions around how child-centred insights should be translated into design specifications need to be further explored in order to streamline the incorporation of children's voices into design interventions. It is hoped that further interdisciplinary work between different stakeholders, practitioners and academics may produce more viable design practices that include and elevate disabled children's voices and are responsive to their desires and ideas.

\section{ACKNOWLEDGMENTS}

The author would like to thank the researchers and IPM stakeholders who contributed to the creation of the child-centred design analysis framework, as well as the Wheels of Change project and the PPL Dream Fund initiative for sharing the secondary data used in this study. Study conduct was supported by the Hugh Greenwood Fund for Children's Health Research.

\section{REFERENCES}

Barroqueiro, D. R. (2000). Art in Early Childhood: An Examination of Form, Content and Social Context. International Art in Early Childhood Research Journal, Volume 2, Number 1.

Can, E. and İnalhan, G. (2017) Having a voice, having a choice: Children's Participation in Educational Space Design, The Design Journal, 20:sup1, S3238-S3251.

Desmet P. and Dijkhuis, E. (2003). A Wheelchair Can Be Fun: A Case of Emotion-driven Design. Designing Pleasurable Products and Interfaces 2003, Pp 22-27.

Hussain, S. (2010). Empowering marginalised children in developing countries through participatory design processes, CoDesign, 6:2, 99-117.

O'Sullivan, C., and Nickpour, F. (2020) 50 Years of Inclusive Design for Childhood Mobility; Insights from an Illustrative Mapping Review. In Boess, S., Cheung, M. and Cain, R. (eds.), Synergy - DRS International Conference 2020, Pp. 332-357.

Oxford. (2016). What is a PESTEL analysis? Oxford College Of Marketing. Available online at: https://blog.oxfordcollegeofmarketing.com/2016/06/30/pestel-analysis/ [accessed 04/09/2020].

Richins, M, L. (1994). Valuing Things: The Public and Private Meanings of Possessions. Journal of Consumer Research, 21 (3), Pp. 504-521.

Robinson, R.E, (2015). 'Building a Useful Research Tool: An Origin Story of AEIOU'. Available online at: https://www.epicpeople.org/building-a-useful-research-tool/ [accessed 04/09/2020].

Spurlock, D. (2020). Scholarship During a Pandemic: Secondary Data Analysis. J Nurs Educ. 59(5).

United Nations (1990). Convention On The Rights Of The Child. UN Commission on Human Rights.

Walter, A. (2011). Hierarchy of User Needs: Designing for Emotion, First Ed. Oct 18, 2011; A Book Apart.

Yamada-rice D. (2019) Including Children in the Design of the Internet of Toys. In: Mascheroni G., Holloway D. (eds) The Internet of Toys. Studies in Childhood and Youth. Palgrave Macmillan, Cham. 